\title{
A Pós-graduAÇÃo NA ESCOLA de ENFERMAGEM de RIBEIRÃo PRETO - USP: EVOLUĢÃO HISTORICA E SUA CONTRIBUIÇÃO PARA O DESENVOLVIMENTO DA ENFERMAGEM
}

\author{
Maria Cecilia Puntel de Almeida ${ }^{1}$ \\ Rosalina Aparecida Partezani Rodrigues ${ }^{2}$ \\ Antonia Regina Ferreira Furegato ${ }^{3}$ \\ Carmen Gracinda Silvan Scochi ${ }^{4}$
}

Almeida MCP, Rodrigues RAP, Furegato ARF, Scochi CGS. A pós-graduação na Escola de Enfermagem de Ribeirão Preto-USP: evolução histórica e sua contribuição para o desenvolvimento da enfermagem. Rev Latino-am Enfermagem 2002 maio-junho; 10(3):276-87.

O trabalho objetiva fazer um resgate dos 25 anos da Pós-Graduação da Escola de Enfermagem de Ribeirão Preto da Universidade de São Paulo, 1975-2000, através de reflexão crítica sobre sua criação e desenvolvimento. A metodologia baseou-se na análise quantitativa da produção da Pós-Graduação (número de alunos, docentes, dissertações e teses defendidas, tempo médio de duração e outros) e em bibliografias sobre o assunto. Os resultados demonstram que a Pós-Graduação se consolidou nos anos 90, criando os níveis de doutorado nos três programas Enfermagem Psiquiátrica, Enfermagem Fundamental e Enfermagem em Saúde Pública. Esse patamar alcançado aponta para a necessidade de projeção internacional.

\section{THE GRADUATE PROGRAMS OFFEREd BY THE UNIVERSITY OF SÃO PAULO AT RIBEIRAOO PRETO COLLEGE OF NURSING: HISTORICAL EVOLUTION AND CONTRIBUTIONS TO NURSING DEVELOPMENT}

This study aims at reviewing the 25 years (1975-2000) since the first graduate program was offered by the University of São Paulo at Ribeirão Preto College of Nursing based on a critical reflection about the creation and development of graduate programs. The methodology applied was a quantitative assessment regarding the scientific production published in the literature about the theme. Results showed that the graduate programs were consolidated in the 1990s, when three doctoral programs were created.: Psychiatric Nursing, Fundamental Nursing and Public Health Nursing. These accomplishments require an increase in international visibility.

DESCRIPTORS: graduate education in nursing, nursing research

\footnotetext{
${ }^{1}$ Professor Titular, Presidente da Comissão de PósGraduação, e-mail: cecilia@eerp.usp.br; ${ }^{2}$ Professor Titular, Coordenadora do Programa de Pós-Graduação em Enfermagem Fundamental, e-mail: rosalina@eerp.usp.br; ${ }^{3}$ Professor Titular, Coordenadora do Programa de Pós-Graduação em Enfermagem Psiquiátrica, e-mail: furegato@eerp.usp.br; ${ }^{4}$ Professor Associado, Coordenadora do Programa de Pós-Graduação de Enfermagem em Saúde Pública, e-mail: cscochi@eerp.usp.br. Escola de Enfermagem de Ribeirão Preto, da Universidade de São Paulo, Centro Colaborador da OMS para o desenvolvimento da pesquisa em enfermagem
} 


\section{EL POSGRAdo EN LA ESCUELA de ENFERMERÍA dE RIBEIRÃo PRETO de LA UNIVERSIDAD DE SAOO PAULO: EVOLUCIÓN HISTORICA Y SU CONTRIBUCIÓN PARA EL dESARROLlo de LA ENFERMERÍA}

Este estudio tiene como objetivo rescatar la historia de los 25 años del Postgrado ofrecido por la Escuela de Enfermería de Ribeirão Preto de la Universidad de São Paulo(1975-2000), a través de una reflexión crítica sobre su creación y desarrollo. La metodología se basó en el análisis cuantitativo de la producción del posgrado (número de alumnos, docentes, tesis y disertaciones defendidas, tiempo promedio de duración y otros) y en bibliografías relacionadas con el tema. Los resultados demostraron que el Posgrado se consolidó en los años 90 con la creación de tres programas del nivel de doctorado: Enfermería Psiquiátrica, Enfermería Fundamental y Enfermería en Salud Pública. El éxito obtenido muestra la necesidad de proyectar este programa a nivel internacional.

DESCRIPTORES: educación de postgrado en enfermería, investigación en enfermería

\section{APRESENTAÇÃO}

$\boldsymbol{O}$ presente trabalho foi elaborado e apresentado no evento de comemoração dos 25 anos do ensino de Pós-Graduação da Escola de Enfermagem de Ribeirão Preto, da Universidade de São Paulo (EERP-USP), com a finalidade de fazer um resgate histórico apontando os momentos mais expressivos, e de analisar qual tem sido sua contribuição para o desenvolvimento da enfermagem. Contou com a colaboração da Assistência Técnica Acadêmica e da Seção de Pós-Graduação dessa Escola.

Para quem já conhece essa história ou dela participou, este texto possibilita a (re)construção do passado em que ela se insere. Para quem não a conheceu ajuda a compreender a conjuntura atual com maior possibilidade de participação, responsabilidade e solidariedade, ligando o passado ao presente e construindo pontes para o futuro.

\section{INTRODUÇÃO}

A Pós-Graduação no Brasil, aprovada pelo Conselho Federal de Educação, em 1965, com base no Parecer Sucupira $n^{\circ}$ 977/65 e instituída pela Reforma Universitária, ocorrida em 1968, além da necessidade de ampliar a capacidade de investigação das universidades e de seu corpo docente, objetivou a formação de professores competentes que pudessem atender à expansão quantitativa do ensino superior, estimular o desenvolvimento da pesquisa por meio de preparação de novos pesquisadores e assegurar o treinamento eficaz de técnicos e trabalhadores intelectuais do mais alto nível, para fazer face às necessidades do desenvolvimento em todos os setores.

É consenso, na comunidade científica nacional, que o ensino da Pós-Graduação é, atualmente, o nível mais bem sucedido. "A PósGraduação é, hoje, um segmento consolidado da educação brasileira. Nas últimas décadas, tem contribuído decisivamente para a formação de recursos humanos qualificados e para o desenvolvimento científico do país, garantindo, no seu âmbito de competência, uma posição de destaque do Brasil, no contexto latinoamericano. A Pós-Graduação desempenha um papel estratégico e constitui, por seu nível de 
excelência, uma das principais condições que possibilitam o aperfeiçoamento do sistema educacional como um todo(1)".

No ano 2000, estavam cadastrados, na CAPES, 1246 cursos de mestrado (41 deles profissionalizantes) e 798 de doutorado. $\mathrm{Na}$ grande área da saúde, há 419 cursos, sendo 214 de mestrado e 205 de doutorado, e a enfermagem que está inserida nessa área conta com 16 Programas de Pós-Graduação, 8 deles com mestrado e doutorado, $7 \mathrm{com}$ apenas mestrado e um com doutorado.

Quanto à localização geopolítica desses Programas da área de enfermagem, no norte e centro-oeste inexiste, no nordeste há 3, no sudeste, 11, e, no sul, 2. No Estado de São Paulo, estão localizados 7 programas (5 com mestrado e doutorado, um só com doutorado e outro com mestrado), conforme mostra a Tabela 1. Maiores detalhes sobre os programas de pósgraduação em enfermagem no Brasil podem ser encontrados no estudo quali-quantitativo ${ }^{(2)}$.

Tabela 1 - Programas de Pós-Graduação em Enfermagem no Brasil, segundo a universidade, o estado e a região de origem. 2000

\section{REGIÄO SUDESTE}

\begin{tabular}{|c|c|c|}
\hline Universidade & Esta do & Programas de Pós-Graduação \\
\hline Universidade de Sẫo Paulo (Sẫo Paulo) & São Paulo & $1(\mathrm{M}$ e $\mathrm{D})$ \\
\hline Universidade de Sẫo Paulo (Ribeirẫo Preto) & São Paulo & $3(\mathrm{M}$ e $\mathrm{D})$ \\
\hline Universidade de São Paulo (Sẫo Paulo/Ribeirẫo Preto) & São Paulo & $1(\mathrm{D})$ \\
\hline Universidade Federal de São Paulo & São Paulo & $1(\mathrm{M} \mathrm{e} \mathrm{D})$ \\
\hline Universidade Estadual de Campinas & São Paulo & $1(\mathrm{M})$ \\
\hline Sub-Total Säo Paulo & & 7 \\
\hline Universidade do Rio de Janeiro & Rio de Janeiro & $1(\mathrm{M}$ e $\mathrm{D})$ \\
\hline UNIRIO & Rio de Janeiro & $1(\mathrm{M})$ \\
\hline Universidade Estadual do Rio de Janeiro & Rio de Janeiro & $1(\mathrm{M})$ \\
\hline Sub-Total Rio de Janeiro & & 3 \\
\hline Universidade Federal de Minas Gerais & Minas Gerais & $1(\mathrm{M})$ \\
\hline Sub-Total Minas Gerais & & 1 \\
\hline TOTAL SUDESTE & & $11(68,75 \%)$ \\
\hline \multicolumn{3}{|c|}{ REGIÄO NORDESTE } \\
\hline Universidade Federal da Paraíba & Paraíba & $1(\mathrm{M})$ \\
\hline Universidade Federal da Bahia & Bahia & $1(\mathrm{M})$ \\
\hline Universidade Federal do Ceará & Ceará & $1(\mathrm{M}$ e $\mathrm{D})$ \\
\hline TOTAL NORDESTE & & $3(18,75 \%)$ \\
\hline \multicolumn{3}{|c|}{ REGIÄO SUL } \\
\hline Universidade Federal de Santa Catarina & Santa Catarina & $1(\mathrm{M}$ e $\mathrm{D})$ \\
\hline Universidade Federal do Rio Grande do Sul & Rio Grande do Sul & $1(\mathrm{M})$ \\
\hline TOTAL SUL & & $2(12,50 \%)$ \\
\hline
\end{tabular}

A EERP-USP contava, no ano 2000, com 4 Programas, sendo 3 com os níveis de mestrado e doutorado (Enfermagem Psiquiátrica, Enfermagem Fundamental e
Enfermagem em Saúde Pública), 1 programa só de doutorado, entre a Escola de Enfermagem de São Paulo e a Escola de Enfermagem de Ribeirão Preto, da 
Universidade de São Paulo (Programa Interunidades de Doutoramento em Enfermagem).

É nesse contexto da pós-graduação brasileira que se objetiva, neste trabalho, fazer uma descrição e reflexão sobre os 25 anos do ensino de pós-graduação na Escola de Enfermagem de Ribeirão Preto, resgatando sua singularidade por meio da criação, desenvolvimento, consolidação e das novas perspectivas a médio prazo que se delineiam.

\section{METODOLOGIA}

A elaboração do presente texto baseouse no levantamento dos dados empíricos quantitativos da série histórica dos 25 anos, 1975 a 2000, relativos ao número de alunos matriculados, número de titulações, tempo médio de titulação, número de docentes, avaliações recebidas dos três Programas de Pós-Graduação da EERP-USP e também do Programa Interunidades de Doutoramento (dados somente da EERP-USP). Utilizou-se também de fontes documentais como relatórios, projetos de criação de cursos e catálogos, principalmente para a transcrição de fatos históricos, relativos ao ensino de pósgraduação na EERP-USP. Outra fonte de dados foi a memória viva de alguns docentes e funcionários que vivenciaram alguns períodos destes 25 anos.

No resgate histórico ora apresentado, considera-se que três períodos merecem destaque: o primeiro corresponde aos últimos anos da década de 60 e início dos anos 70 , quando ocorreu a qualificação de um grupo líder de docentes e a criação do primeiro curso de mestrado em Enfermagem Psiquiátrica, seguido pelo de Enfermagem Fundamental; o segundo período, nos anos 80 , caracteriza a luta pela criação do nível de Doutorado, e o terceiro período, década de 90 , representa a consolidação da Pós-Graduação e dos Núcleos de Pesquisa, sem deixar de apontar os novos desafios que já estão colocados.

\section{PERÍODOS HISTÓRICOS}

$1^{\circ}$ Período: final dos anos 60 e década de $70-$ Preparando/qualificando o corpo docente para a Pós-Graduação

Nesse período, ainda era possível obter o título de doutor sem a necessidade de cursar a Pós-Graduação, como hoje está instituída. $O$ corpo docente da EERP-USP contava, em seu quadro, com uma socióloga, Célia Almeida Ferreira Santos, que organizou, juntamente com o Departamento de Matemática Aplicada à Biologia da Faculdade de Medicina de Ribeirão Preto-USP, um curso de pósgraduação de longa duração - "Bases da Experimentação em Enfermagem". Este foi ministrado pelo então chefe do Departamento, Prof. Dr. Geraldo Garcia Duarte, no segundo semestre de 1967 e primeiro de 1968. O grupo de docentes que participou do curso, teve a oportunidade de elaborar seus projetos de pesquisa, origem das primeiras teses de doutorado. Assim, as primeiras docentes que obtiveram o título de doutor foram: Célia Almeida Ferreira Santos, Maria Aparecida Minzoni, Maria Helena Machado, Maria Cecília Manzolli, Emília Luigia Saporiti Angerami, Nilza Tereza Rotter Pelá, Judith Costa e Vera Heloisa Pileggi Vinha. Dessa forma, nos primeiros anos da década de 70 , estava qualificado (grau de 
doutor) esse primeiro grupo de docentes que iria liderar a pesquisa e a organização da PósGraduação na EERP-USP.

Até então, a Escola estava estruturada em dois departamentos: o de Enfermagem Geral e Especializada e o de Enfermagem Psiquiátrica e Ciências Humanas. Este segundo saiu à frente com o Curso de Mestrado em Enfermagem Psiquiátrica, em 1975, o terceiro curso de Pós-Graduação stricto sensu em enfermagem a ser criado no país após a reforma universitária, que se deu em 1968.

A Profa. Dra. Maria Aparecida Minzoni foi a enfermeira psiquiátrica que liderou esse curso, com a colaboração e participação de outros líderes docentes. Mesmo sendo da área de concentração em enfermagem psiquiátrica, o curso, que, no ano de 1980, foi credenciado junto ao Conselho Federal de Educação, aceitou alunos de outras especialidades da enfermagem. "O programa em nível de mestrado - área Enfermagem Psiquiátrica, teve, na sua primeira matrícula, 30 alunos, a maioria dos quais, por força das circunstâncias, eram docentes da Escola de Enfermagem de Ribeirão Preto e pertenciam a outras especialidades que não a Enfermagem Psiquiátrica. Isso se deu porque a USP exigia um tempo limitado para que os docentes estivessem matriculados em um curso de pósgraduação, sem o que seriam rescindidos seus contratos como docentes"(3).

O Departamento de Enfermagem Geral e Especializada deu início, em 1979, ao Curso de Mestrado em Enfermagem Fundamental, tendo como fio condutor a assistência clínica de enfermagem e o cuidado em saúde à pessoa adulta. $2^{\circ}$ Período: anos 80 - A luta para a criação do doutorado

Com uma experiência de alguns anos na formação de mestres, adquirindo vivência na pesquisa, e considerando a não existência do nível doutorado na enfermagem brasileira, um grupo de docentes da EERP-USP começou a colocar em ação estratégias para a criação de um curso de doutorado. Foram muitas idas e vindas, travando-se um trabalho intenso, de corpo a corpo, com várias instâncias da Universidade.

As dificuldades foram bem retratadas por uma reflexão crítica sobre os limites impostos pela comunidade científica hegemônica, na incorporação de grupos emergentes ${ }^{(4)}$.

Em 1979, a EERP-USP e a Escola de Enfermagem da Universidade de São Paulo (EE-USP) entraram, separadamente, na Câmara de Pós-Graduação da Universidade de São Paulo, com a solicitação de curso de doutorado. "Inicialmente, houve informações, por parte de um dos membros do Conselho Universitário, de que a Câmara de PósGraduação considerava as duas Escolas sem massa crítica para oferecer, isoladamente, o doutorado e que a única forma de tornar o doutoramento possível seria o oferecimento de um programa conjunto. A partir dessa informação, os membros das duas Comissões de Pós-Graduação (CPG) passaram a discutir a forma de reunir as idéias para elaborar um projeto conjunto"(5).

A Comissão de seis membros, composta pelas presidentes das duas CPG (EE-USP e EERP-USP) e mais duas docentes de cada Escola, foi assim constituída: Wanda de Aguiar Horta, Amália Corrêa de Carvalho, Evalda Cançado Arantes, Maria Helena Machado, 
Maria Aparecida Minzoni e Nilza Tereza Rotter Pelá ${ }^{(5)}$. A mesma autora informa que "o período de trabalho foi de outubro de 1979 a abril de 1981 com incontáveis reuniões que exigiam deslocamento constante de docentes para realizarem o trabalho".

$$
\text { Em maio de 1981, o Programa }
$$
Interunidades de Doutoramento em Enfermagem foi aprovado pela Câmara de PósGraduação da USP e credenciado pelo Conselho Federal de Educação, em 1986.

Os diretores das duas Escolas designaram membros para compor a Comissão Interunidades de Pós-Graduação (CIPG), que passou a gerenciar esse Programa. Como orientadores, além de docentes da própria Escola, foram também incluídos docentes de outras unidades da USP, principalmente da Faculdade de Medicina de Ribeirão Preto.

Outro fato marcante que contribuiu para alavancar a formação de pesquisadores da EERP-USP foi a sua designação em 1988 como Centro Colaborador da Organização Mundial da Saúde para o desenvolvimento da pesquisa em enfermagem. Assim projetou-se a necessidade de qualificação do corpo docente no nível de doutorado, a curto prazo ${ }^{(6-7)}$.

$3^{\circ}$ Período: anos 90 - Consolidando a PósGraduação e traçando estratégias futuras

Em 26 de maio de 1985, iniciou-se o desdobramento do Departamento de Enfermagem Geral e Especializada da EERPUSP, criando-se o Departamento de Enfermagem Materno-Infantil e Saúde Pública (Resolução 2960, de 20 de fevereiro de 1986). Disciplinas que já guardavam afinidade entre si puderam agregar-se, constituindo uma nova área de conhecimento.
Desde então, a EERP-USP está constituída por três Departamentos que abrangem, no conjunto, a totalidade dos conhecimentos da enfermagem.

Em 1991, foi criado o terceiro curso da EERP-USP, o de Mestrado de Enfermagem em Saúde Pública, no Departamento de Enfermagem Materno-Infantil e Saúde Pública. Esse curso é multiprofissional e interdisciplinar, tendo a atenção primária em saúde, como fundamentação, e a saúde coletiva, como fio condutor.

No mesmo ano de 1991, o Departamento de Enfermagem Geral e Especializada solicitou a extensão do Mestrado de Enfermagem Fundamental para o Doutorado, o qual foi aprovado e iniciou seu funcionamento com o oferecimento de 5 vagas.

Em 1996, portanto, a EERP-USP só contava com o Doutorado no Programa de Enfermagem Fundamental e no Programa Interunidades de Doutoramento em Enfermagem (Interunidades) sendo, este último, comum às duas Escolas de Enfermagem da Universidade de São Paulo. Os Departamentos de Enfermagem Psiquiátrica e Ciências Humanas e de Enfermagem Materno-Infantil e Saúde Pública, que tinham os Cursos de Mestrado, estavam preparados e desejavam estender seus mestrados também para doutorado. Assim, organizou-se uma Comissão para estudar a reestruturação da Pós-Graduação da Escola de Enfermagem como um todo, objetivando otimização do quadro de orientadores e da infra-estrutura disponível. Uma das propostas era ter somente um Programa de Pós-Graduação com três áreas de concentração, mestrado e doutorado, em cada um dos Departamentos.

A proposta de reestruturação não foi 
aceita pela maioria dos docentes, mantendose a tendência histórica de se criarem, em cada Departamento, seus próprios programas de pós-graduação. Assim, os dois Departamentos procuraram organizar as propostas de extensão de seus Mestrados para os Doutorados.

Em 1998, o Departamento de Enfermagem Materno-Infantil e Saúde Pública estendeu seu mestrado para o doutorado e, em 1999, isso ocorreu com o Departamento de Enfermagem Psiquiátrica e Ciências Humanas. Portanto, somente na década de 90, é que, de fato, a Pós-Graduação na EERP se consolidou, quando os três Departamentos passaram a contar com os dois níveis de formação (mestrado e doutorado), constituindo os atuais Programas de Pós-Graduação.

Na década de 90, o ensino universitário no país passou por graves dificuldades estruturais devido ao ajuste econômico do país e à reforma do Estado e da Previdência Social com as privatizações, enxugamento do quadro de funcionários e aposentadoria precoce de grande número de docentes universitários. Nas universidades públicas federais, havia o incentivo salarial para os que detinham o grau de Doutor. "Esta situação conjuntural do ensino público em nível superior repercutiu de maneira mais acentuada na enfermagem, pois naqueles 20 anos de pós-graduação (1970-1990) não houve uma política deliberada de formação do corpo docente em pós-graduação na enfermagem, principalmente no nível de doutorado. Quase todos os doutores em enfermagem concentravam-se na região sudeste. Somente no início da década de 90 houve uma grande mobilização das Escolas de Enfermagem para a qualificação de seu corpo docente. Assim, surgiram, de várias regiões do país, solicitações aos cursos da região sudeste, de assessoria para a implantação da pósgraduação. Aquelas instituições que já tinham uma certa infra-estrutura solicitaram a abertura da pós-graduação, nível mestrado. Outras, que não tinham doutores em seus quadros, reuniam esforços e discutiam projetos integrados com várias universidades na modalidade de consórcio ou rede de pós-graduação”(8).

A EERP-USP, com seus Programas de Pós-Graduação consolidados, foi muito solicitada, e ainda continua sendo, para realizar convênios com outras instituições, a fim de fazer a formação de doutores. Firmaram-se convênios/acordos institucionais com as seguintes instituições:

- Universidade Federal da Bahia (março de 1995), com titulação de 4 doutores;

- Universidade Federal da Paraíba (março de 1995), com titulação de 4 doutores;

- Universidad de Concepción - Chile (março de 1996), com titulação de 4 doutores e

- Universidade Federal do Rio Grande do Norte (março de 1997), com titulação de 6 doutores.

Alunos de outros países da América Latina, como Chile, Colômbia, Peru, Argentina, e também de Angola - Continente Africano, têm procurado titulação nos Programas da EERPUSP, com apoio financeiro das instituições de origem ou pelo Programa Estudante Convênio PEC/PG, da Fundação Coordenação de Aperfeiçoamento de Pessoal de Nível Superior - CAPES, para alunos estrangeiros.

No final dos anos 90 e durante o ano de 2000, com a criação do Mestrado Interinstitucional (MINTER) pela CAPES, os Programas da Escola de Enfermagem têm sido solicitados por outras Instituições de Ensino Superior, para elaborar esse tipo de convênio. Já foi realizado um convênio MINTER (19981999), através do Programa de Enfermagem 
Fundamental, tendo, como instituição receptora, a Universidade do Oeste do Paraná - Departamento de Enfermagem do campus de Cascavel. Foram titulados 11 mestres. Outros MINTER encontram-se em negociação, bem como outros convênios com outros estados brasileiros, no sentido de contribuir para a formação de doutores. Estão em andamento as negociações com o México, para elaboração de um convênio que propiciará a formação de enfermeiros doutores para aquele país.

A consolidação da Pós-Graduação na Escola de Enfermagem está evidenciada nas tabelas e gráficos apresentados a seguir.
A Figura 1 e a Tabela 2 mostram o início dos cursos, a evolução do número de alunos regularmente matriculados nos Programas, até $o$ ano de 2000. Percebe-se o aumento gradativo do número de matrículas ano a ano, desde 1975, ao longo dos 25 anos. Observa-se que é na década de 90, que, de fato, a PósGraduação se consolida, pois conta-se com o nível de mestrado e doutorado nos três Programas. Quanto ao número de alunos, há uma curva ascendente bastante acentuada; em 1980, contava-se com 41 alunos, em 1990, com 101, em 1995, com 198 e, em 2000, conta-se com 288 pós-graduandos.

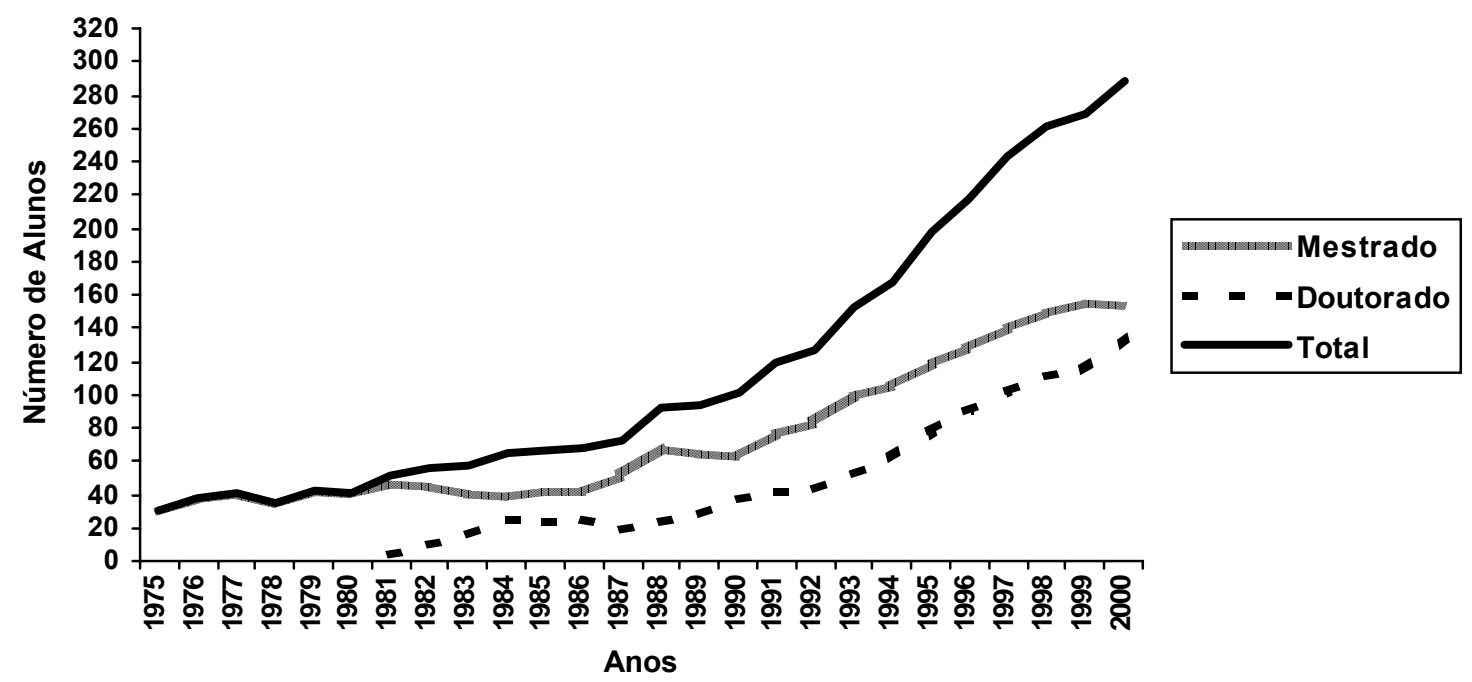

Figura 1 - Número de alunos de mestrado e doutorado matriculados nos Programas de PósGraduação da Escola de Enfermagem de Ribeirão Preto, da Universidade de São Paulo. 19752000

Tabela 2 - Programas de Pós-Graduação da Escola de Enfermagem de Ribeirão Preto, da Universidade de São Paulo, segundo o ano de início e número de alunos regularmente matriculados no ano de 2000

\begin{tabular}{lccccc}
\hline \multirow{2}{*}{ Programas de Pós-Graduação } & \multicolumn{2}{c}{ Ano de Início } & \multicolumn{2}{c}{$\mathbf{N}^{\circ}$ de Alunos/2000 } & \multirow{2}{*}{ Total } \\
& Mestrado & Doutorado & Mestrado & Doutorado & \\
\hline Enfermagem Psiquiátrica & 1975 & 1999 & 34 & 17 & 51 \\
Enfermagem Fundamental & 1979 & 1991 & 66 & 46 & 112 \\
Enfermagem em Saúde Pública & 1991 & 1998 & 54 & 27 & 81 \\
Doutorado Interunidades & - & 1981 & - & 44 & 44 \\
TOTAL & & & $\mathbf{1 5 4}$ & $\mathbf{1 3 4}$ & $\mathbf{2 8 8}$ \\
\hline
\end{tabular}


É preciso que se diga que, enquanto, no ensino de graduação da EERP-USP, há 320 alunos matriculados, e o número de vagas (80 ao ano) é constante há alguns anos, na pósgraduação, o número de alunos vem crescendo acentuadamente, principalmente nos últimos 5 anos, atendendo a demanda da região e país. Esse aumento anual, crescente, de alunos na pós-graduação vem ocorrendo em todas as áreas da Universidade de São Paulo.

A demanda de alunos, até meados dos anos 80 , era constituída, em grande parte, por docentes de instituições públicas de ensino superior e uma porcentagem menor por enfermeiros dos serviços de saúde. Atualmente, está em crescimento a demanda de enfermeiros de serviços e de docentes de instituições privadas, possivelmente devido à exigência de titulação mínima de mestre para a docência universitária, segundo a Lei de Diretrizes e Bases do Ensino.

Espera-se, ainda, um aumento do número de vagas, nos próximos anos. $A$ capacidade da pós-graduação ainda não está no seu limite em relação ao número de docentes credenciados e ao número de orientandos por orientador, como pode ser visto na Tabela 3. Porém, sabe-se que não são somente esses parâmetros quantitativos que determinam o número de vagas, mas outros, como, por exemplo, o compromisso social da universidade na formação de pesquisadores para regiões carentes do país.

Tabela 3 - Número de docentes credenciados e de alunos regularmente matriculados nos Programas de Pós-Graduação da Escola de Enfermagem de Ribeirão Preto, da Universidade de São Paulo. Setembro/2000

\begin{tabular}{|c|c|c|c|c|c|c|c|}
\hline \multirow[t]{2}{*}{ Programas } & \multicolumn{3}{|c|}{$\mathbf{N}^{\circ}$ de Docentes credenciados } & \multicolumn{3}{|c|}{$\mathbf{N}^{\circ}$ de Alunos matriculados } & \multirow{2}{*}{$\begin{array}{l}\text { Relação Orientador/ } \\
\text { Orientando }\end{array}$} \\
\hline & M & $M / D$ & TOTAL & M & D & TOTAL & \\
\hline Enfermagem Psiquiátrica & 3 & 11 & 14 & 34 & 17 & 51 & 3,6 \\
\hline Enfermagem Fundamental & 14 & 19 & 33 & 66 & 46 & 112 & 3,4 \\
\hline Enfermagem Saúde Pública & 11 & 14 & 25 & 54 & 27 & 81 & 3,2 \\
\hline Doutorado Interunidades & - & 31 & 31 & - & 44 & 44 & 1,4 \\
\hline TOTAL & & & & 154 & 134 & 288 & \\
\hline
\end{tabular}

O número de dissertações e teses defendidas também cresce em curva ascendente, conforme Tabela 4 e Figura 2. Nesses 25 anos, foram titulados 525 pósgraduandos, sendo 376 mestres e 149 doutores. A média anual de defesas, nos cinco períodos analisados, tem aumentado acentuadamente, correspondendo a 5,4 (M) 9,0 ( $M$ e D), 15,2 ( $M$ e D), 28,6 ( $M$ e D) e 58,5 ( $\mathrm{M} \mathrm{e}$ D), respectivamente.

Tabela 4 - Número de defesas de mestrado e doutorado dos Programas de Pós-Graduação da Escola de Enfermagem de Ribeirão Preto, da Universidade de São Paulo. 1977 a 2000

\begin{tabular}{lcccccc}
\hline PeríodoDefesas & $\mathbf{1 9 7 7 - 1 9 8 1}$ & $\mathbf{1 9 8 2 - 1 9 8 6}$ & $\mathbf{1 9 8 7 - 1 9 9 1}$ & $\mathbf{1 9 9 2 - 1 9 9 6}$ & $\mathbf{1 9 9 7 - 2 0 0 0}$ & Total Geral \\
\hline Mestrado & 27 & 33 & 55 & 101 & 160 & 376 \\
Doutorado & - & 12 & 21 & 42 & 74 & $\mathbf{1 4 9}$ \\
Total & 27 & 45 & 76 & 143 & 234 & $\mathbf{5 2 5}$ \\
\hline
\end{tabular}




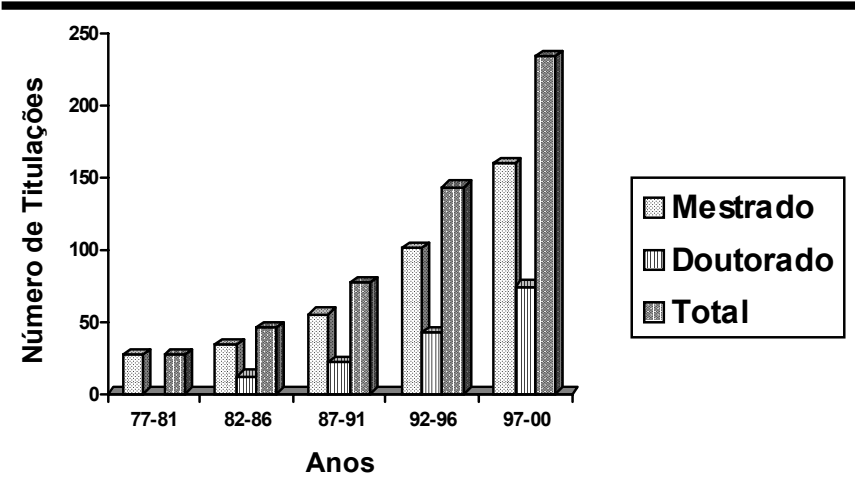

Figura 2 - Número de titulações de mestrado e doutorado dos Programas de Pós-Graduação da Escola de Enfermagem de Ribeirão Preto, da Universidade de São Paulo. Período de 1977 a 2000

Quanto às avaliações da CAPES, no último biênio (1996-1997), a maioria dos Programas obteve nota 5, que corresponde ao conceito Bom. As notas menores ( 3 e 4 ) devemse ao fato de que os cursos de doutorado estão iniciando. As notas do sistema de avaliação da CAPES variam de 2 a 7 , sendo que são reconhecidos pelo MEC os programas com nota igual ou maior de 3 . O critério das notas 6 e 7 é para o curso de excelência e que tem projeção internacional. Nenhum programa de enfermagem do país obteve, até então, nota acima de 5. Estes dados estão na Tabela 5.

Tabela 5 - Avaliação da CAPES referente aos Programas de Pós-Graduação da Escola de Enfermagem de Ribeirão Preto, da Universidade de São Paulo. Biênio 1996/1997

\begin{tabular}{lcc}
\hline \multicolumn{1}{c}{ Programas } & \multicolumn{2}{c}{ Biênio 96/97 } \\
& Mestrado & Doutorado \\
\hline Enfermagem Psiquiátrica & 5 & 3 \\
Enfermagem Fundamental & 5 & 5 \\
Enfermagem Saúde Pública & 5 & 4 \\
Doutorado Interunidades & - & 5 \\
\hline
\end{tabular}

Fonte: Capes $^{(9)}$
Considera-se que alcançar um nível de excelência é um dos desafios que já estão colocados para os próximos 5 anos e, para tal, é imprescindível intensificar a produção científica internacional e em periódicos da área indexados e reconhecidos pelos pares, bem como a realização de pós-doutorado no exterior, para que ocorra a projeção internacional da pesquisa.

A porcentagem de evasão dos mestrados nos Programas da EERP-USP, segundo dados da Pró-Reitoria de PósGraduação-USP, é de 5\%, no período de 1994 a 1998, sendo um dos mais baixos, não só na área da saúde, como também nas demais áreas do conhecimento.

O tempo de conclusão dos mestrados, ao longo dos 25 anos, vem diminuindo conforme mostra a Tabela 6. No triênio 1997/ 1999, foi de 39 meses, tempo ainda elevado, segundo critérios da CAPES, que estipula 24 meses. No doutorado, o tempo de titulação aumentou no período de 1987 a 1991, a partir daí decresceu, chegando a 42 meses, o que é considerado bom.

Tabela 6 - Tempo de conclusão (em meses) dos mestrados e doutorados dos Programas de Pós-Graduação da Escola de Enfermagem de Ribeirão Preto, da Universidade de São Paulo. 1977 a 1999

Período/ 1977-1981 1982-1986 1987-1991 1992-1996 1997-1999 Cursos

\begin{tabular}{lccccc}
\hline Mestrado & 56,5 & 58,5 & 56,9 & 40,2 & 39 \\
Doutorado & - & 41,3 & 57,2 & 49,1 & 42 \\
\hline
\end{tabular}

\section{CONSIDERAÇÕES FINAIS}

A consolidação do ensino de PósGraduação foi um dos fatores determinantes 
do desenvolvimento da enfermagem brasileira.

Na EERP-USP, a Pós-Graduação produziu impacto considerável na produção científica da área, permitindo elucidar várias lacunas do conhecimento da enfermagem e aprofundar muitas outras temáticas, garantindo, dessa forma, o seu espaço junto aos pares da comunidade científica.

Esse novo conhecimento tem sido utilizado no ensino de graduação, renovandoo e atualizando-o, bem como na assistência de enfermagem e no setor saúde de forma geral, quando se tem oportunidade de melhorar a assistência de enfermagem prestada à população, promovendo-se a qualidade de vida. A produção de conhecimento gerada na PósGraduação tem contribuído para uma efetiva articulação entre a universidade e a sociedade, contemplando uma variedade de contextos da prática de enfermagem, desde a promoção da saúde, prevenção de doenças, tratamentos, reabilitação até o cuidado hospitalar de maior complexidade.

Atender a demanda crescente de alunos de várias localidades e, principalmente, de regiões carentes de Pós-Graduação tem possibilitado formar lideranças em pesquisa para essas regiões.

Outros impactos do ensino de pósgraduação da EERP/USP e que vêm contribuindo para a enfermagem brasileira são: - consolidação dos grupos e núcleos de pesquisa na enfermagem;

- definição e consolidação das linhas de pesquisa;

- intercâmbio nacional e internacional de conhecimento científico de enfermagem com professores e pesquisadores;

- maior visibilidade da profissão de enfermagem no setor saúde e na sociedade;

- conquista de espaço participativo nas instituições científicas e tecnológicas (Conselho Nacional de Desenvolvimento Científico e Tecnológico - CNPq, Fundação Coordenação de Aperfeiçoamento de Pessoal de Nível Superior - CAPES, Fundação de Amparo à Pesquisa do Estado de São Paulo - FAPESP, Sociedade Brasileira para o Progresso da Ciência - SBPC, etc.).

Como o conhecimento é um processo cumulativo e contínuo, alguns desafios já estão colocados para atingir-se o nível de excelência e projeção internacional. O volume da produção científica, nos próximos 5 anos, tem que ser intensificado, devendo ser divulgada, também, em periódicos indexados internacionais. Intensificar o intercâmbio internacional dos pesquisadores brasileiros de enfermagem é também outro desafio colocado para os Programas de Pós-Graduação da EERP-USP.

Uma das estratégias para alcançar o nível de projeção internacional é o desenvolvimento de centros de excelência, aglutinando-se pesquisadores em torno das prioridades de pesquisa em enfermagem, formando-se células que, em futuro próximo, poderão reproduzir-se, constituindo-se sistemas consolidados em pesquisa. 


\section{REFERÊNCIAS BIBLIOGRÁFICAS}

1. Mec/Capes (BR). Infocapes: edição comemorativa dos 45 anos. Boletim Informativo da CAPES; 1996.

2. Moriya TM, Rodrigues ARF, Almeida MCP, Ruffino MC, Oliveira MHP. Pós-graduação stricto sensu em Enfermagem: um estudo do seu desenvolvimento no Brasil. Ribeirão Preto (SP): FIERP - Fundação Instituto de Enfermagem de Ribeirão Preto; 1998.

3. Pelá NTR. O ensino de enfermagem na Escola de Enfermagem de Ribeirão Preto: o ensino de pósgraduação. In: Universidade de São Paulo. Livro Comemorativo do $30^{\circ}$ aniversário da Escola de Enfermagem de Ribeirão Preto da Universidade de São Paulo. Ribeirão Preto (SP): USP; 1985. p.62-4.

4. Almeida MCP, Barreira IA. Estudos de pós-graduação em enfermagem na América Latina: sua inserção na comunidade científica. In: Sena RR. Educación de Enfermería en América Latina. Bogotá: Universidad Nacional; 2000. p.129-45.

5. Arantes EC. Histórico do Programa Interunidades de Pós-Graduação em Enfermagem. In: Universidade de São Paulo. Programa Interunidades de Pós-Graduação - 1982-1992. São Paulo (SP): USP; 1992. p.5-16.

6. Mendes IAC, Trevizan MA. Ações do Centro Colaborador da OMS para o desenvolvimento da pesquisa em enfermagem: o caso brasileiro. Acta Paulista Enfermagem 2000 agosto; 13(2):9-15.

7. Mendes IAC, Gir E, Trevizan MA. Escola de Enfermagem de Ribeirão Preto da Universidade de São Paulo: Centro Colaborador da Organização Mundial da Saúde. Rev Latino-am Enfermagem 1993 dezembro; 1 ( ${ }^{\circ}$ especial): 53-68.

8. Gutiérrez MGR, Vieira TT, Almeida MCP, Elsen I, Stefanelli MC Acompanhamento e avaliação da pósgraduação no Brasil: retrospectiva histórica da representação da enfermagem. Escola de Enfermagem Anna Nery Revista Enfermagem 2001 agosto; 5(2):16172.

9. Capes (BR) [online] 1999, nov. Disponível na Internet.URL: http://www.capes.gov.br 